\title{
Isolated Fractures of the Sustentaculum Tali: Injury Characteristics and Surgical Technique for Reduction Management
}

\author{
${ }^{1} \mathrm{Hu} \mathrm{Mu},{ }^{2} \mathrm{Xu}$ Xiangyang, ${ }^{3} \mathrm{Bi}$ Gang
}

\begin{abstract}
Isolated fractures of sustentaculum tali are rare and often missed at an initial visit. They can be difficult to manage and may have implications for future hindfoot function. This article describes sustentacular fracture patterns and characteristics, and a method of surgical treatment via a medial approach to the calcaneus. During the last 6 years, 11 recent isolated sustentacular fractures without calcaneal posterior facet involvement were surgically-treated at our hospital. Of these all underwent open reduction and internal fixation via medial approach to the calcaneus. Patient charts, X-ray radiographs and CT scans were reviewed for injury characteristics and associated musculoskeletal injures. Five patients had intra-articular middle facet involvement, and two had subtalar joint subluxation. By cannulated screws reduction was maintained through fractures rigidly fixation. Isolated fracture of the sustentaculum tali is tend to be ignored and easily misdiagnosed as acute ankle sprain. Open reduction and internal fixation of this fractures was reliable and should be procedure via medial approach.
\end{abstract}

Keywords: Sustentaculum tali, Calcaneus, Fracture, Open reduction and internal fixation, Foot injuries.

How to cite this article: Mu H, Xiangyang X, Gang B. Isolated Fractures of the Sustentaculum Tali: Injury Characteristics and Surgical Technique for Reduction Management. J Foot Ankle Surg (Asia-Pacific) 2014;1(2):48-51.

Source of support: Nil

Conflict of interest: None

\section{INTRODUCTION}

The annual incidence of calcaneus fractures is below $1.2 \%$. ${ }^{1,2}$ Isolated fractures of the sustentaculum tali are very rare. ${ }^{3}$ They have been regarded as extra-articular fractures, despite the fact that displaced fractures invariably lead to incongruity of the articular surface of the subtalar joint. Tarsal tunnel syndrome can be caused by some severe injury. Few published articles exist and most publications are limited to case reports. ${ }^{4}$ The sustentaculum tali includes the middle facet of the

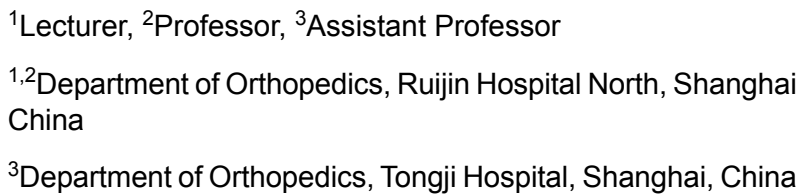

Corresponding Author: Xu Xiangyang, Professor, Department of Orthopedics, Ruijin Hospital North, Shanghai, China, Phone: 862164370045, e-mail: xu664531@hotmail.com subtalar joint and contiguous with the anterior facet of the subtalar joint occasionally. The sustentaculum serves as an attachment point of the deltoid ligament and of the spring ligament. The flexor hallucis longus tendon (FHL) sits in a groove beneath the sustentaculum. Fractures of the sustentaculum tali can lead to incongruity of extraarticular surfaces, possibly compromising stability of the transverse tarsal joint and of the subtalar joint. Malunited sustentacular fractures can affect the FHL tendon seriously leading to subtalar arthrosis and hindfoot stiffness, as same as other intra-articular fractures years after. All in all, we recommend open reduction and internal fixation for isolated fractures with those patients with tarsal tunnel syndrome or articular displacement of more than $1 \mathrm{~mm} .^{5}$

The indications and the best method of surgical treatment have not been established due to the rarity of the fracture and the paucity of literature. Since, the sustentaculum tali located under the medial malleolus, classical extensile L-shaped lateral approach to the calcaneus and reduction as described by zwipp can be very difficult. Only medial approach allows for the display of the cortical fracture line and the adjacent posterior tibia neurovascular bundle directly. ${ }^{6}$ The purpose of this article is to report the fracture morphology, medial surgical approach for operation and fixation of displaced sustentacular fractures. We report a series of recent isolated sustentaculum tali fractures of the calcaneus treated by open reduction and internal fixation. The mechanism of this rare injury as well as the indications and techniques of open reduction and internal fixation are discussed.

\section{PATIENTS AND METHODS}

From September 2006 to March 2011, 11 patients with isolated sustentaculum tali fractures of the cancalneus were treated by open reduction and internal fixation at our department. All 11 patients had a recent fractures of the sustentaculum tali. At the time of follow-up, weight-bearing and standard radiographs were taken. The orthopedic trauma association/AO classification of fractures was used, with patients being screened for inclusion on the basis of a classification of OTA/AO types 73-B fractures (partial articular calcaneus fractures) and 
73-C fractures (complete articular calcaneus fractures). Radiographs were reviewed to determine the fracture classification. Eleven were identified as isolated fractures of the sustentaculum tali and were treated via a medial approach to the calcaneus. All 11 patients were evaluated using the American Orthopaedic Foot and Ankle Society (AOFAS) standardized clinical scoring system for the hindfoot. The results were rated as excellent with 90 to 100 points, good with 80 to 89 , fair with 70 to 79 or poor with $<70$ points.

The average age of the 11 patients in the study group was 26 (17-41) years. Seven patients sustained right-sided fractures and four sustained left-sided fractures. Mechanisms of injury included a motor vehicle crash in three, a fall from a substantial height in five, a pedestrian struck by motor vehicle in three. All patients underwent operative management of their fractures by the same surgical team. All patients had basic demographic characteristics recorded, such as age, side of injury (right versus left), mechanism of injury, and associated other musculoskeletal injuries. X-ray and CT scans, as well as clinical notes, were reviewed for fracture patterns.

\section{SURGICAL INTERVENTION}

Surgery was performed under lumbar or epidural anesthesia. The patient was placed in a supine position with slightly external rotation of the limb and facilitate approach to the medial malleolus. A pneumatic tourniquet was applied at the thigh and the surgical field was prepared leaving the leg exposed. For the medial approach to reduction and fixation of the sustentacular fractures, a oblique incision is made right parallel to the path of the posterior tibial tendon sheath beneath the medial malleolus. The posterior tibial tendon (PT) sheath is then incised, flexor digitorum longus (FDL) and the PT retracted dorsally, the neurovascular bundle (posterior tibial arterial, PTA and posterior tibial nerve, PTV), plantar and posterior to the approach, is retracted plantarly and protected. This Interval between the PT and the PTA allows access to the inferior cortical exit point of the fracture. Fixation is typically accomplished with 1 or 2 cannulated screw or mini-plates as necessary (Figs 1A to F).

\section{RESULTS}

Scrutiny of injury radiographs and CT scans yielded a description of fracture characteristics. Five patients were noted to have widening of the calcaneus, as assessed on axial radiography. Six patients were noted to have subtalar dislocations associated with the sustentacular fracture; in five of these patients, articular comminution was present, and in two articular depression was present.
All patients underwent open reduction and internal fixation of their sustentacular fractures at an average of 6.5 days after injury (5-9 days). Eleven patients were treated exclusively through a medial approach, canulated screw fixation medially. No wound complications were noted. Seven of the 11 patients had radiographic followup of an average of 14.3 (4-36) months. The mean time to bone union was 8.5 weeks (8-16 weeks). Four patients lost to follow-up at our center. All seven patients exhibited radiographic healing at a minimum of 3 months. Results rated using the hindfoot scale (100 points total) according to AOFAS. The outcome of the recent fractures was Excellent in five and good in two. One fracture developed superficial infection, which resolved with vacuum drainage and oral antibiotic therapy.

\section{DISCUSSION}

1. Symptom characteristics and the differential diagnosis of the fractures of sustentaculum tali: Isolated sustentacular fractures of the calcaneus are relatively rare, as illustrated by the paucity of reports available in the literature. In spite of this, they do occur, and little has been written about their characteristics and surgical treatment skills.

The patient complains the pain around the medial malleolus after falling or traffic accident, but most patients are not lost the ability of walking completely. Patient often walks in a special antalgic gait (forefoot pronation, plantar flexion, hindfoot varus), only walking in this gait. The pain can be alleviated or even disappeared to patients. Therefore, the antalgic gait is the most remarkable characteristic of this type of fracture different from other fractures. ${ }^{6}$ Tenderness can be found in the medial malleolus obviously by physical checking, with or without subcutaneous ecchymosis in the medial malleolus.

2. Biomechanics: As sustentacular fractures have historically been considered to be extra-articular fractures, conservative management such like plaster fixation has been advocated, especially in the treatment of minimal displacement of the fractures. Also, the FHL tendon may become interposed within the upper fracture line, bring mechanical incarceration and perhaps bone nonunion. ${ }^{3}$ Tarsal tunnel syndrome has also been reported with hypertrophy of the sustentaculum tali after nonunion of a sustentacular fracture. ${ }^{7}$ Only anatomical reduction and rigid fixation can help to prevent these late sequelae happening. Biomechanical studies have been performed to describe the relative contribution of the middle facet to the total weight bearing forces seen by the subtalar joint during normal ambulation. Wagner et $\mathrm{al}^{8}$ 

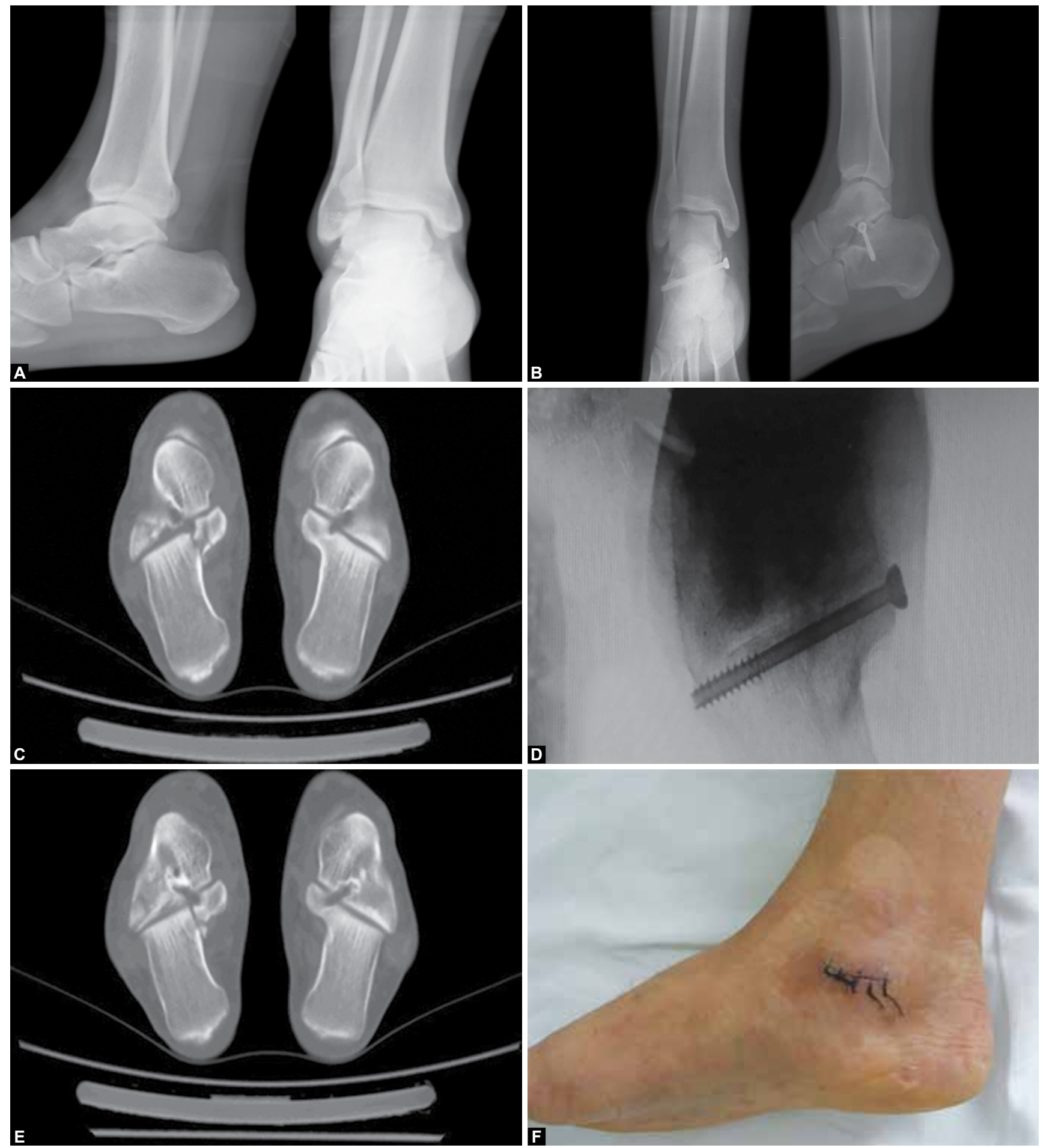

Figs 1A to F: Male, 38 years old, fallen from 2 meters high. Isolated fractures of the right sustentaculum tali: (A) radiograph showing the isolated fractures of the right sustentaculum tali, $(B$ and $C)$ CT scan showing the isolated fractures of the right sustentaculum tali, $(D$ and $E$ ) radiographs showing the restoration of the sustentaculum tali by cannulated screw postoperative and $(F)$ medial wound 2 weeks after operation

performed a biomechanical cadaveric study on load distribution between the middle facet and the posterior facet of the subtalar joint. They found that the contact area for the middle facets was only $31 \%$ of the total area for the subtalar joint, despite the load being carried by the middle facets equaling $63 \%$ of that of the subtalar joint. Mean contact pressures were 1.44 MPa for the middle facets and 0.93 MPa for the posterior facet. This data indicate that the middle facet sees disproportionately high loads as pressure increases across the ankle and hindfoot. Sangeorzan et $\mathrm{al}^{9}$ demonstrated that contact areas across the posterior facet were significantly decreased with calcaneus fracture displacement of $2 \mathrm{~mm}$ or more, leading to the suggestion that this degree of displacement of posterior facet fractures should be 
addressed with operative reduction. With knowledge of the increased contact pressures and small surface area for the middle facet of the subtalar joint, it seems reasonable also to suggest that small displacements of sustentacular fractures perhaps should be treated surgically. Obtaining anatomical reduction in this location can yield a good indirect reduction of the articular surface of subtalar joint.

3. Surgical approaches: As the sustentaculum tali locates right beneath the medial malleolus, the classical L-shaped lateral extensile approach to the calcaneus does not provide visualization of the fracture fragments directly. Via a lateral approach, fracture lines can be obstructed by the intact calcaneal tuberosity. ${ }^{10}$ So, we strongly recommend the medial approaches for sustentacular fractures that involves direct reduction of fracture fragments.

Medial approaches to the calcaneus have been described. McReynolds ${ }^{11}$ initially introduced a medial approach for reduction and fixation of calcaneus fractures. Zwipp et $\mathrm{al}^{12}$ also described medial approach in over two-third of cases for open reduction and internal fixation of displaced intra-articular calcaneus fractures, he emphasizing the sustentacular fragment as the 'key block' to reduction. Burdeaux ${ }^{13}$ then subsequently published a review of 61 calcaneus fractures treated through a medial approach at a mean follow-up of 4.4 years, and demonstrated good-to-excellent results in 46 patients, as assessed with the AOFAS scoring system. If the main, or the only, fracture line exits medially, and restoration of the sustentacular fragment to the sustentacular fragment is the goal, then a medial approach provides excellent display of the medial cortex.

This study has several limitations, including the small number of patients treated and the lack of longterm follow-up. The small number of patients is a testament to the infrequency of this injury. We only collect 11 patients that underwent open reduction and internal fixation of isolated sustentacular fractures during the 6-year collection period. Gatha et $\mathrm{a}^{14}$ reported only four cases from the 15-year practice of a single trauma surgeon. Although we have limited follow-up data and can draw few conclusions in this kind of fractures, the rarity of the injury coupled with the regional nature of our trauma center makes obtaining follow-up exceedingly difficult. A multicenter, prospective cohort study would likely be required for a more in-depth exploration of outcomes of patients who sustain isolated sustentacular fractures of the calcaneus.

\section{REFERENCES}

1. Sanders R. Displaced intra-articular fractures of the calcaneus. J Bone Joint Surg Am 2000;82(2):225-250.

2. Rammelt $S$, Zwipp H. Calcaneus fractures: facts, controversies and recent developments. Injury 2004;35(5):443-461.

3. Romash M. Fracture of the calcaneus: an unusual fracture pattern with subtalar joint interposition of the flexor hallucis longus. A report of two cases. Foot Ankle Int 1992;13(1):32-41.

4. Myerson MS, Berger BI. Nonunion of a fracture of the sustentaculum tali causing a tarsal tunnel syndrome: a care report. Foot Ankle Int 1995;16(11):740-742.

5. Garchar DJ, Lewis JE, DiDomenico LA. Hypertrophic sustentaculum tali causing a tarsal tunnel syndrome: a case report. J Foot Ankle Surg 2001;40(2):110-112.

6. Della Rocca GJ, Nork SE, Barei DP, et al. Fractures of the sustentaculum tali injury characteristics and surgical technique for reduction. Foot Ankle Int 2009;30(11):1037-1041.

7. Myerson MS, Berger BI. Nonunion of a fracture of the sustentaculum tali causing a tarsal tunnel syndrome: a care report. Foot Ankle Int 1995;16(11):740-742.

8. Wagner UA, Sangeorzan BJ, Harrington RM, et al. Contact characteristics of the subtalar joint: load distribution between the anterior and posterior facets. J Orthop Res 1992;10(4):535-543.

9. Sangeorzan BJ, Ananthakrishnan D, Tencer AF. Contact characteristics of the subtalar joint after a simulated calcaneus fracture. J Orthop Trauma 1995;9(3):251-258.

10. Burdeaux BD Jr. The medial approach for calcaneal fractures. Clin Orthop 1993;290:96-107.

11. McReynolds IS. The case for operative treatment of fractures of the os calcis. In Leach RE, Hoaglund FT, Riseborough EJ. Controversies in Orthopaedic Surgery, Philadelphia, Saunders; 1982.

12. Zwipp H, Tscherne H, Thermann H, et al. Osteosynthesis of displaced intra-articular fractures of the calcaneus: results in 123 cases. Clin Orthop 1993;290:76-86.

13. Burdeaux BD Jr. Fractures of the calcaneus: open reduction and internal fixation from the medial side a 21-year prospective study. Foot Ankle Int 1997;18(11):685-692.

14. Gatha M, Pedersen B, Buckley R. Fractures of the sustentaculum tali of the calcaneus: a case report. Foot Ankle Int 2008;29(2):237-240. 